\title{
Visualizador cartográfico y construcción de bases de información sobre infraestructura eléctrica en Centroamérica ${ }^{\hat{k}}$
}

\section{Cartographic display and construction of information database on electric infrastructure in Central America.}

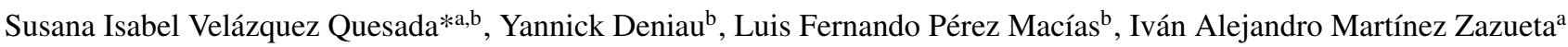 \\ ${ }^{a}$ Universidad Nacional Autónoma de México, Mexico City, 04510, Mexico. \\ ${ }^{b}$ GeoComunes: http://geocomunes.org/
}

\section{Resumen}

En este trabajo se presenta la construcción y visualización de bases de información exhaustivas sobre el sistema eléctrico de Centroamérica que comprende las centrales de generación eléctrica (priorizando las mayores a 5 MW), las líneas de transmisión, así como algunos megaproyectos directamente relacionados. Diseñada y desarrollada por el Colectivo Geocomunes con apoyo de organizaciones sociales, este mapeo y base de datos funge como un punto de partida de un proyecto de cartografía colaborativa. Su objetivo es proporcionar una base de información que fortalezca la capacidad de las comunidades locales y de la población en general para discutir, argumentar, monitorear y exigir la rendición de cuentas en el sector eléctrico de la región. Una capacidad social que consideramos necesaria frente al contexto de privatización de bienes comunes estratégicos como lo es la electricidad, en donde el Estado ha cedido al mercado global su facultad de gestión y toma de decisiones.

Keywords: Electricidad; Megaproyectos; Energías Renovables; Defensa del territorio; Cartografía colaborativa

\begin{abstract}
In this work we present the construction and visualization of an exhaustive spatial database of the electrical power infrastructure of Central America, which include generation plants greater than $5 \mathrm{MW}$, transmission lines, and some associated megaprojects. Designed and developed by the Geocomunes Collective in collaboration with social organization, this cartography and its associated database are the starting point for a collaborative mapping project. The objective of this research is to provide an information database that aims at strengthen the capacity of local communities in Central America and the population in general to discuss, argue, monitor, and demand accountability mechanisms. A social capability that we consider highly needed in a global context of privatization of strategic common goods, such as power supplies, where the State has ceded to the global market its capacities of management and decision making
\end{abstract}

Palabras clave: Electricity generation; Megaprojects; Renewable energy; Defense of the territory; Collaborative mapping

(c) S. I. Velázquez-Quesada, Y. Deniau, L. F. Pérez-Macías, I. A. MartínezZazueta.

This is an Open Access article distributed under the terms of the Creative Commons Attribution License (https://creativecommons.org/licenses/by-nc-sa/4.0/), which permits non-commencial sharing of the work and adaptions, provided the original work is properly cited and the new creations are licensed under identical terms.

*E-mail address: geocomunes@gmail .com

\section{Introducción}

\section{1. Área de investigación y su contexto}

La investigación se hizo sobre países que forman parte del Sistema de Interconexión Eléctrica para América Central (SIEPAC): Guatemala, Honduras, El Salvador, Nicaragua, Costa Rica y Panamá; y rastreando principalmente las centrales de generación eléctrica mayores a $5 \mathrm{MW}$.

Desde la década de los noventa, Centroamérica vive un proceso de expansión de infraestructura para la generación y transmisión de electricidad y, particularmente desde 2010, de proyectos asociados con una diversificación de fuentes de energías 
renovables. A pesar de que esta es una región donde la cobertura de este servicio no ha llegado a toda la población y donde los montos de la importación de energía primaria fósil resultan costosos para las finanzas públicas; la expansión de la capacidad de producción eléctrica con fuentes renovables no ha sido bien vista por las poblaciones de estos países. El despliegue de esta infraestructura ha despertado luchas y oposiciones de comunidades que no ven en la proliferación de centrales eléctricas la realización de un bien de utilidad e interés público, sino la imposición del interés privado de empresas locales y sobre todo extranjeras sobre territorios comunitarios. ${ }^{1}$ La participación del capital privado en el subsector eléctrico de la región se legalizó bajo distintas modalidades en todos los países desde mediados de los noventa, ${ }^{2}$ y desde entonces estas empresas han sido consideradas como el principal motor de la electrificación de la región (con excepción de Costa Rica, donde el capital del Estado sigue siendo prioritario en el desarrollo de esta infraestructura). En la mayoría de los países, la privatización del sector ocurrió de forma paralela a la configuración de Mercados Mayoristas ${ }^{3}$ que operaban en escalas nacionales. Esto es, mercados de electricidad donde los generadores privados podrían vender electricidad directamente a los grandes consumidores (sean estos transmisoras o distribuidoras de electricidad) y que funcionan como mecanismos que garantizan el consumo o la venta de la electricidad producida. En este contexto, la puesta en operación del Mercado de Electricidad Regional (MER) un mercado que opera de manera paralela a los mercados nacionales, y del SIEPAC, que es la red de transmisión eléctrica que posibilita estas transferencias de energía, no sólo ha dinamizado la inversión privada, sino que ha incrementado el número de proyectos eléctricos. La relación entre la instalación de este mercado regional y la expansión de proyectos eléctricos en la región ha sido expuesta en otro documento de análisis del colectivo (Geocomunes, 2019a). En este artículo, el objetivo principal es mostrar la construcción de bases de datos georreferenciadas sobre estos proyectos eléctricos, los principales resultados de este mapeo y las posibilidades que ofrece su visualización.

En este contexto, observamos que:

- Pese al crecimiento de la capacidad de generación eléctrica en la región, no existen bases de datos públicas que

\footnotetext{
${ }^{1}$ Citamos a modo de ejemplo de esta oposición social, la resistencia en Costa Rica contra el proyecto de hidroeléctrica Diquís, hoy cancelado; la oposición al proyecto Hidroeléctrico el Cimarrón en El Salvador; la oposición del pueblo Ngabe Buglé en Panamá contra la construcción de la presa Changuinola II; el conflicto por el Proyecto hidroeléctrico Tumarín en Nicaragua; la oposición al proyecto Hidroeléctrico Patuca III en Honduras, y la oposición contra el proyecto hidroeléctrico Xalalá en Guatemala (ver Enviromental Justice Atlas, 2019). Aunque todas tienen particularidades, estas oposiciones y cuestionamientos explícitos a los proyectos de generación eléctrica están centrados en la defensa de la integridad territorial comunitaria.

${ }^{2}$ En Guatemala esta apertura ocurrió en 1996; en Costa Rica en 1995; en Nicaragua en 1998; Honduras en 1994, El Salvador en 1996; y en Panamá en 1997.

${ }^{3}$ Nuevamente, el único país perteneciente al SIEPAC que al día de hoy no tiene la figura de Mercado Mayorista es Costa Rica.
}

estén completas y actualizadas sobre las centrales de generación eléctrica en operación, construcción y en proyecto a escala nacional ni a escala regional. La información a la que se puede acceder no está georeferenciada y cuando llega a estarlo, no se encuentra actualizada y está incompleta o con errores en la georeferenciación ${ }^{4}$.

- No existe información clara y sistematizada sobre los capitales relacionados con las centrales que se tienen en proyecto. Información básica que debería estar al alcance de toda la población para poder llevar a cabo un ejercicio de monitoreo y de exigencia de rendición de cuentas en tanto usuarios del sistema.

- En los documentos oficiales, los nombres de las empresas indicadas como promoventes o titulares de las centrales eléctricas suelen hacer referencia a filiales y no a las empresas matrices. Un hecho que dificulta de forma importante el análisis de datos sobre la centralización en el sector eléctrico aún a sectores especializados.

- Existe una ausencia de información sobre los bancos públicos y privados que financian los proyectos, así como de los mecanismos a través de los cuales estos financiamientos tienen lugar.

Ante este contexto de falta de información sobre el sistema eléctrico existente y en proyecto, los actores que lo controlan o aquellos que lo financian, la investigación que presentamos aporta una base de información exhaustiva sobre la infraestructura y los actores asociados directamente a ella. Una investigación que busca ser el punto de partida de un monitoreo colaborativo que provea al público en general (académicos, comunidades y pueblo interesado) de información actualizada sobre este sector estratégico.

Es en relación con esto último, que un elemento central de la investigación lo constituye el visualizador que permite la difusión, la actualización e intervención permanente de esta base de información, un elemento que potencia enormemente los mapas estáticos que se difundieron como parte de este proyecto.

A diferencia de mapas estáticos, que se diseñaron y difundieron con el fin de que esta investigación llegue directamente a las comunidades afectadas por la construcción de la infraestructura eléctrica, muchas de las cuales no cuentan con energía eléctrica o no son cercanas al uso de herramientas digitales ni del internet; este visualizador está diseñado para ser un medio de comunicación y colaboración con comunidades más urbanizadas y con grupos de investigación y acompañamiento a comunidades rurales.

El mapa interactivo se presenta en el sitio http://geocomunes. org/Visualizadores/Centroamerica/. Tiene componentes y funcionalidades que representan ventajas significativas sobre otros

\footnotetext{
${ }^{4}$ Una excepción notable es el caso de El Salvador, donde sí existe una base georreferenciada para los proyectos de energías renovables.
} 
proyectos de geovisualización referentes a conflictos socioambientales y/o megaproyectos que atentan contra los bienes comunes. Una de estas características la constituye la posibilidad de consultar el texto de análisis de la investigación en el que se incluyen gráficas interactivas y tablas relativas al mismo. Así mismo, otro hito alcanzado es la funcionalidad que permite consultar de forma dinámica las tablas de proyectos de generación de electricidad, así como las empresas y financiamientos relacionados, permitiendo descomponer la base de datos para su análisis. Además, otro componente fundamental es que permite la descarga tanto de las capas de información a través de Geonode, como de los materiales en versión "imprimible" producto de la investigación. Elementos que fueron diseñados para hacer de este visualizador una herramienta de consulta, de descarga de información y de actualización colaborativa de la información por elemento o región cartografiada.

En este sentido, el mapa digital que presentamos resulta distinto de otros similares que existen en el ámbito de la justicia ambiental y el monitoreo de conflictos como, por ejemplo, el Environmental Justice Atlas (EjAtlas) de la Institut de Ciència i Tecnologia Ambientals - Universidad Autónoma de Barcelona. En tanto este último se centra en la presentación de un mapa con el despliegue global de puntos sobre conflictos socioambientales clasificados por tipo de conflicto, la descarga de la documentación de los mismos y de otras capas relacionadas, así como una funcionalidad de filtros avanzados con base en los parámetros sistematizados sobre los casos geolocalizados; el que presentamos con esta investigación busca ser una plataforma de visualización de información detallada sobre el sistema eléctrico, de descarga de información sobre cada una de los proyectos, y de comprensión del sistema eléctrico así como de formación metodológica para realizar un monitoreo sobre este sistema (sección que está detallada en el material escrito que también puede ser consultado en el visualizador). Este visualizador está enfocado en el mapeo preciso del conjunto de proyectos que conforman la infraestructura eléctrica existente y proyectada en la región y no pretende aportar información sobre los conflictos derivados de la instalación de esos proyectos, como lo hace por ejemplo el EJAtlas.

\subsection{Objetivo de la investigación}

Construir una base de datos georeferenciada exhaustiva sobre las centrales de generación eléctrica en operación, construcción y en proyecto en los países que forman parte del SIEPAC, así como un mapa interactivo en línea para su consulta, descarga y análisis. La base de datos está ampliamente detallada en diversas cualidades de las centrales, como son datos de tecnología empleada, de la capacidad de generación (en MW), de la empresa promovente, la empresa matriz, el capital al que pertenece la infraestructura (público o privado) y la inversión desembolsada para su planeación y construcción (millones de dólares).

A través del mapa interactivo en línea se proporciona información completa y de acceso público sobre la infraestructura eléctrica en esta región que coadyuve a la discusión, la toma de decisiones y la exigencia de rendición de cuentas de la población frente a temas relacionados con el sistema eléctrico y sobre la utilidad pública de algunos de los proyectos de infraestructura energética.

Esta investigación busca tener impacto sobre tres tipos de público:

a. Comunidades directamente afectadas por el desarrollo de proyectos de generación eléctrica y organizaciones acompañantes de éstas;

b. Población en general, principalmente de la región centroamericana, preocupada por el sentido social, ambiental y culturalmente adecuado de la electricidad y;

c. Público especializado en el tema energético que podrá hacer uso de la base para desarrollar análisis diversos.

En este sentido, el mapa dinámico publicado en internet y de acceso público, constituye una plataforma para que esta investigación y las bases de datos que la sostienen (principalmente archivos en formato shapefile) puedan ser consultadas, descargadas y, eventualmente, actualizadas en equipos colaborativos que procedan atendiendo a la metodología de búsqueda ahí explicitada.

\section{Metodología}

\subsection{Construcción y publicación de las bases de datos}

Las bases de datos se construyeron en tres etapas, priorizando siempre el uso de fuentes de información oficial (pública y privada), y complementando y cotejando con fuentes de información hemerográfica de medios especializados y locales.

\subsubsection{Construcción de una lista completa de las centrales de generación eléctrica}

La primera fase de la construcción de las capas georeferenciadas consistió en revisar las listas de centrales eléctricas en operación, construcción y proyecto que proporcionan las distintas instancias oficiales en cada país y principalmente los autoridades en materia eléctrica, encargadas de dar autorización a los proyectos $^{5}$.También se revisaron listas de otras fuentes como las publicadas en los Anuarios Eléctricos de la Comisión Económica para América Latina y el Caribe (CEPAL), para luego hacer una suma de todas las centrales mencionadas.

Si bien estas listas oficiales de centrales suelen contabilizar todas y cada una de las plantas conectadas al Sistema Eléctrico

\footnotetext{
${ }^{5}$ Las fuentes revisadas para cada país son las siguientes: Costa Rica: Autoridad Reguladora de los Servicios Públicos (ARESEP); Instituto Costarricense de Electricidad; Guatemala: Ministerio de Energía y Minas (MEM); El Salvador: Superintendencia General de Electricidad y Telecomunicaciones (SIGET); Honduras: Secretaria de Energia Recursos Naturales Ambiente y Minas (SERNA); Comisión Reguladora de Energía Eléctrica (CREE); ProHonduras; Nicaragua: Ministerio de Energía y Minas (MEM); Comisión Nacional de Energía; Panamá: Autoridad Nacional de los Servicios Públicos (ASEP); Empresa de Transmisión Eléctrica Panameña; Ministerio de Ambiente.
} 
Nacional, se limitan a proporcionar los datos más básicos sobre cada central: nombre, fase, capacidad en MW, tipo de tecnología y ubicación general (municipio o departamento); en ningún caso de los países mencionados estas listas contienen las coordenadas de ubicación exacta de cada central e inclusive, llegan a presentar errores e inconsistencias en la información.

Esta primera fase nos permitió tener el número de centrales por nombre, tipo de tecnología (hidroeléctrica, solar, eólica, geotérmica, térmica fósil y biomasa), fase (en operación, en construcción y en proyecto), así como la capacidad de las centrales a georeferenciar.

\subsubsection{Geolocalización de esas listas de proyectos}

Para geolocalizar las centrales identificadas se hizo una búsqueda de las coordenadas correspondientes a cada una de éstas. La fuente principal de información para esto fueron las diferentes instancias energéticas y ambientales que en cada país tienen competencia sobre el subsector eléctrico.

En algunos casos las instancias gubernamentales anteriormente mencionadas ofrecen en línea una ficha descriptiva de las centrales donde aparecen las coordenadas o un mapa de ubicación. Sin embargo, esas fichas no existen para todos los países ni para todas las centrales, en general se limitan a las centrales en operación, en construcción o los proyectos más grandes. Para ubicar todas las demás centrales revisamos los permisos oficiales que requiere cada proyecto. Uno de esos permisos es la Manifestación de Impacto Ambiental (MIA), también llamado Estudio de Impacto Ambiental (EIA) en algunos países ${ }^{6}$.

Sin embargo, el parámetro a partir del cual los países exigen para la construcción y operación de una central eléctrica la

\footnotetext{
${ }^{6}$ Las fuentes consultadas por país para este tipo de información fueron para Costa Rica: Ministerio de Ambiente y la Energía (MINAE); Guatemala: el Ministerio de Ambiente y Recursos Naturales (MARN); El Salvador: Ministerio de Medio Ambiente y Recursos Naturales (MARN); Nicaragua: Ministerio del Ambiente y de los Recursos Naturales (MARENA); Honduras: Dirección General de Evaluación y Control Ambiental; y para Panamá: Autoridad Nacional del Ambiente de Panamá (ANAM).
}

elaboración de una MIA o EIA varía y no es obligatoria en todos los casos. Un problema que se suma a esta situación es que algunas MIAs o EIAs no son de acceso público, por ejemplo en Honduras la información relativa a las licencias ambientales se declaró como información reservada en 2018 (Ver: Diario Oficial de la República de Honduras, del 17 de Agosto de 2018). Para ubicar el resto de centrales que se encuentran en el mapa, usamos otras fuentes de información, principalmente la gaceta del Diario Oficial de la Federación de cada país dónde se publican los contratos o permisos así como la resolución definitiva de estos trámites. Para algunas centrales no logramos encontrar coordenadas exactas y la ubicación se hizo a partir de imágenes y mapas oficiales donde se señalaba la ubicación de la central en cuestión. Una vez que obtuvimos la lista completa de centrales georeferenciadas hicimos una verificación de la ubicación a partir de imágenes satelitales para las centrales en operación o en construcción.

\subsubsection{Construcción de las tablas de atributos}

La tercera parte de la construcción de la información georeferenciada consistió en elaborar una tabla de atributos lo más exhaustiva posible. A la información general que proporcionan las listas oficiales fuimos agregando nuevas columnas de atributos que nos parecían relevantes sobre todo con la intención de documentar los capitales involucrados. A continuación se muestra el detalle de la tabla de atributos construida con las fuentes de información para cada campo (ver Tabla 1). Cabe mencionar que no se logró obtener la información completa de todos los campos para todas las centrales mapeadas debido a los problemas anteriormente señalados.

\subsubsection{Desarrollo y publicación de Geovisualizador}

Un siguiente paso realizado fue el desarrollo de un geovisualizador en línea, el cual consiste en una aplicación web con un mapa interactivo que permite consultar las capas de información georreferenciada, así como información estadística con gráficas interactivas y tablas de búsqueda. También permite la

Tabla 1: Tabla de atributos de la capa de centrales de generación eléctrica.

Table 1. Attribute table of the power generation plant layer.

\begin{tabular}{ll}
\hline Campo & Fuentes y metodología \\
\hline Nombre & Listas oficiales de instancias gubernamentales \\
Fase & Listas oficiales de instancias gubernamentales y notas de prensa para su actualización \\
Filial & Listas oficiales de instancias gubernamentales \\
Matriz & Páginas oficiales de las empresas, documentos oficiales de compra venta y contratos, notas de prensa \\
Sector & Listas oficiales de instancias gubernamentales \\
Empresa constructora & Páginas oficiales de las empresas y de notas de prensa \\
Empresa proveedora de tecnología & Páginas oficiales de las empresas y de notas de prensa \\
Capacidad en MW & Listas oficiales de instancias gubernamentales \\
Inversión & Páginas oficiales de las empresas y bancos nacionales e internacionales, notas de prensa \\
Financiadores & Páginas oficiales de los principales bancos de desarrollo que operan en la región \\
Provincia / departamento & Listas oficiales de instancias gubernamentales \\
País & Listas oficiales de instancias gubernamentales \\
Conexión al SIEPAC & Informes de la Empresa Operadora de la Red (2016 y 2017) \\
Precisión & Metodología de geolocalización del punto: por coordenadas oficiales; o por referencias oficiales (dirección) y vi- \\
& sualización del elemento. \\
\hline
\end{tabular}


consulta del texto de análisis producto de la investigación. Para ello, en primer lugar, se publicó en un servidor de mapas en línea la base de datos de información geográfica descrita en los puntos anteriores. En segundo lugar, se desarrolló el visualizador web utilizando herramientas de software de código abierto, añadiendo las funcionalidades de activación/desactivación de capas de información geográfica; identificación de objetos geoespaciales y despliegue de resultados en tabla; consulta de texto de análisis; consulta y búsqueda de lista de proyectos de centrales energéticas y funcionalidad para seleccionar desde tabla de resultados las centrales desplegadas en el mapa; consulta de gráficas interactivas con la información generada; consulta y búsqueda de lista de financiamientos; consulta y búsqueda de lista de empresas; y sección de descargas de las capas de información y de materiales en versión para imprimir relativos a la investigación. Una capa que fue tratada de forma especial dentro del visualizador fue la de proyectos de generación eléctrica, la cual consta de dos visualizaciones: a) de acuerdo al tipo de tecnología y fase (proyecto, construcción, operación, cancelada) y b) con tamaño basado en la capacidad productiva y tipo de tecnología. El geovisualizador también permite identificar gráficamente las centrales que pertenecen al SIEPAC.

El geovisualizador está basado en una arquitectura clienteservidor, compuesta en su totalidad por software libre. Como servidor de mapas se utilizó Geoserver conectado a una base de datos PostgreSQL con la extensión espacial PostGIS. Para gestionar los contenidos geoespaciales del proyecto (capas, estilos, tablas) se incluyó la plataforma Geonode. La aplicación web del geovisualizador se publicó en un Apache HTTP Server con un entorno PHP y fue desarrollada utilizando principalmente las librerías javascript OpenLayers, para la implementación del mapa interactivo, D3.js, para el despliegue de gráficas interactivas y DataTables, para incluir las tablas y búsquedas de información. Geoserver funciona como el componente central para la consulta de información geográfica, tanto de los objetos espaciales desplegados en el mapa interactivo, como de las consultas de tablas de atributos y la descarga de capas en distintos formatos. El conjunto de la tecnología empleada está basada en software de código abierto y en estándares de la Open Geospatial Consortium (OGC), lo que permite la interoperabilidad con otras tecnologías dentro de los Sistemas de Información Geográfica y la World Wide Web (WWW).

\subsection{Descripción de los datos encontrados}

La información incluida en la base de datos georreferenciada, permite hacer una descripción sobre aspectos cualitativos y cuantitativos de los proyectos eléctricos en la región (ver Figura 1). En total, en esta investigación se lograron geolocalizar 745 centrales (378 en operación, 57 en construcción y 310 en proyecto). En su conjunto, estas centrales eléctricas sumaban para el año 2018, una capacidad total en operación de aproximadamente 16,876.7 MW. Si a esta cantidad le agregamos lo que

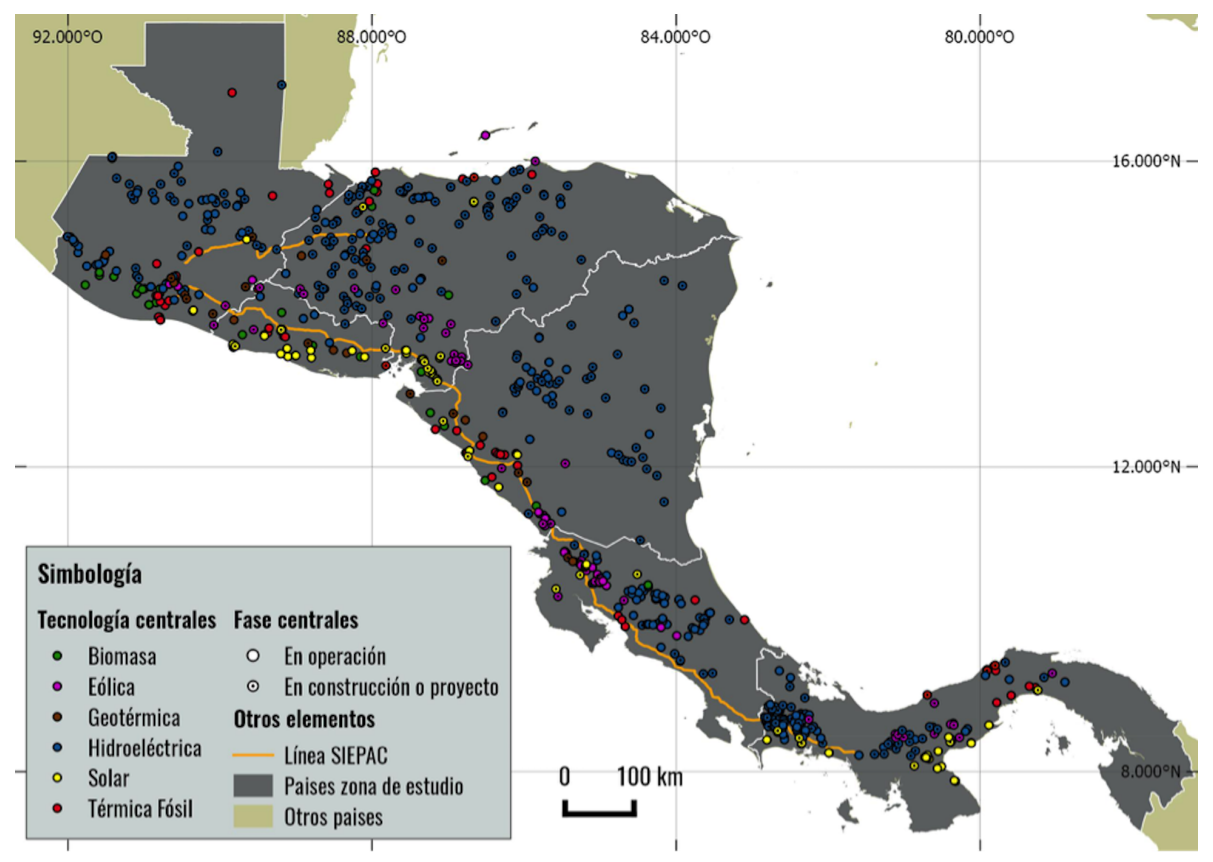

Figura 1. La figura muestra el mapa de la zona de estudio y de las centrales eléctricas georreferenciadas, de acuerdo a su tecnología y energía primaria empleada

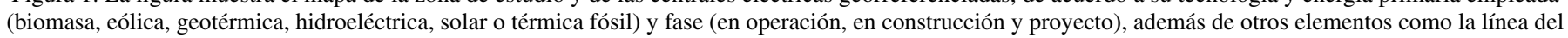
Sistema de Interconexión Eléctrica para América Central (SIEPAC) y la delimitación estatal de los países en cuestión. Fuente: Elaboración propia con base en capas de la investigación.

Figure 1. Geographic distribution of power plants in Central America. The map shows country delimitation, the Central American Electrical Interconnection System (SIEPAC), and the power plants according with their technology: biomass, wind, geothermal, hydroelectric, solar or fossil-fuel; and the project status: projected, in construction or operating. Source: Prepared by the authors based on the georeferenced databases of this research. 
actualmente se está construyendo, la capacidad instalada suma 19,000 MW.

Analizando la distribución de esta infraestructura por país, tenemos que Honduras y Panamá son los países con mayor número de centrales en cualquiera de sus fases (207 y 201, respectivamente); mientras El Salvador y Nicaragua los que menos tienen (50 y 101 respectivamente). Mientras tanto, en Costa Rica y Guatemala se encontraron, respectivamente, 109 y 129 proyectos en cualquiera de sus fases.

En la Figura 2 puede verse el desglose por fase y tecnología de las centrales reportadas en esta investigación. De esta información resulta interesante señalar la concentración en la fase de proyecto, y su contraste con el número de centrales en construcción.

Otro atributo de la base de datos es la capacidad de cada proyecto de generación y la tecnología empleada. Con esta información se puede visualizar a distintas escalas la distribución de los proyectos y su capacidad eléctrica, tal como se muestra en la Figura 3 para el caso de Honduras.

Si bien la infraestructura de generación eléctrica que opera con fuentes de energía renovable tiene una espacialidad acotada por la existencia de los recursos naturales que las sostienen (ríos, viento, etc.), la distribución de estos proyectos puede mostrar patrones importantes para el análisis, como zonas de concentración de la capacidad eléctrica, zonas sin capacidad, distribución de los proyectos por escala de producción, etc.

La figura 4 muestra la repartición de centrales entre el sector público y privado. Con la excepción de Costa Rica, resulta claro que la producción de energía eléctrica está en manos del sector privado, ya que éste sector tiene el $79 \%$ del número de centrales operando, el $95 \%$ de centrales en proyecto, el $70 \%$ de la capacidad instalada, el $93 \%$ de la capacidad proyectada y el $81 \%$ de la generación eléctrica actual.

De acuerdo con esta investigación, las empresas que concentraron más capacidad eléctrica instalada en 2017 son las que pueden visualizarse en la Tabla 2, junto con el país donde están dadas de alta.

En la Tabla 3 se muestra la síntesis de la información que se logró ubicar en esta investigación, sobre los financiamientos a proyectos eléctricos, de los tres principales bancos multilaterales de desarrollo que operan en la región.

Todos los resultados presentados en esta parte por medio de mapas estáticos y tablas, están disponibles de manera dinámica en el visualizador desarrollado (Geocomunes, 2019a). Así

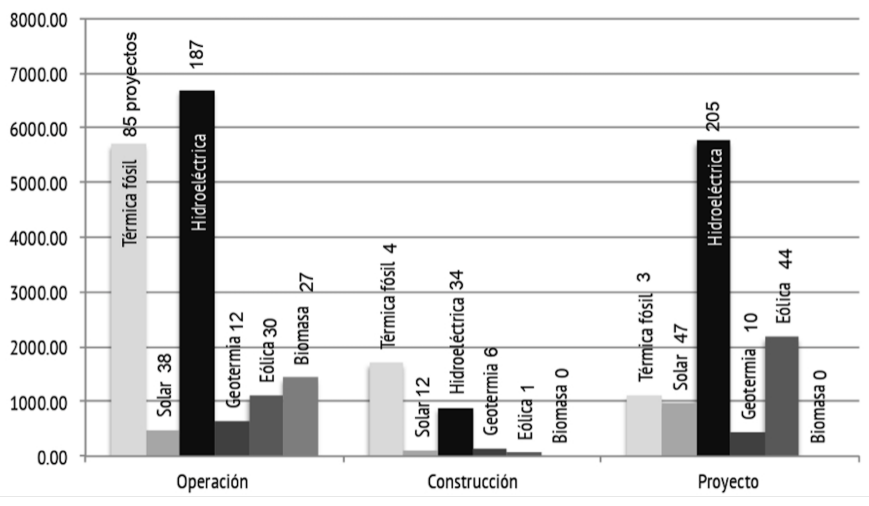

Figura 2. Gráfica de la capacidad instalada total en mega watts (MW) por fase de cada tecnología y con el dato del número de proyectos mapeados en esta investigación. Fuente: Elaboración propia con base en capas de la investigación.

Figure 2. Total power plant capacity in megawatts (MW) in Central America. The graph shows the total power-plant capacity by technology and project status. Source: Prepared by the authors based on the databases of this research.

Tabla 2: Empresas con la mayor capacidad de generación eléctrica instalada en los países del SIEPAC, en el año 2017. Se incluye país de la empresa, el sector (público o privado), así como la capacidad total instalada por firma y lo que esto representa en porcentaje de toda la capacidad instalada conectada al SIEPAC. Fuente: Elaboración propia con base en la base de datos georreferenciada de esta investigación.

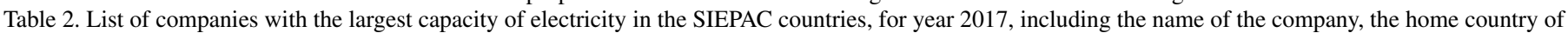
the company, the sector (public or private), the total capacity in megawatts (MW) and the percentage of this capacity being connected to the SIEPAC. Source: Prepared by the authors based on the georeferenced databases of this research.

\begin{tabular}{|c|c|c|c|c|c|}
\hline & Empresa & País de la empresa & Sector & $\begin{array}{c}\text { Capacidad Total } \\
\text { (MW) }\end{array}$ & $\begin{array}{l}\text { \% de la capa- } \\
\text { cidad conecta- } \\
\text { da al SIEPAC }\end{array}$ \\
\hline 1 & Grupo ICE & Costa Rica & Público & 2,662 & 93 \\
\hline 2 & I Squared Capital & EEUU & Privado & 1,064 & 50 \\
\hline 3 & Grupo CEL & Ecuador & Público & 794 & 97 \\
\hline 4 & Grupo Terra & Honduras & Privado & 784 & 84 \\
\hline 5 & AES Corporation & EEUU & Privado & 780 & 99 \\
\hline 6 & Empresa Nicaragüense de Electricidad & Nicaragua & Público & 667 & 81 \\
\hline 7 & Enel Green Power & Italia & Privado & 589 & 82 \\
\hline 8 & LUFUSSA & Honduras & Privado & 509 & 92 \\
\hline 9 & Instituto Nacional de Electrificación (INE) & Guatemala & Público & 475 & 63 \\
\hline 10 & PDVSA y Petronic & Nicaragua y Venezuela & Privado & 472 & 96 \\
\hline 11 & Grupo Argos & Colombia & Privado & 416 & 88 \\
\hline 12 & Ingenio Magdalena & Guatemala & Privado & 303 & 36 \\
\hline 13 & Ashmore Energy International Ltd. & Islas Caimán & Privado & 300 & 100 \\
\hline 14 & InterEnergy & Islas Caimán & Privado & 268 & 100 \\
\hline 15 & Corporación Multi Inversiones & Guatemala & Privado & 262 & 94 \\
\hline
\end{tabular}




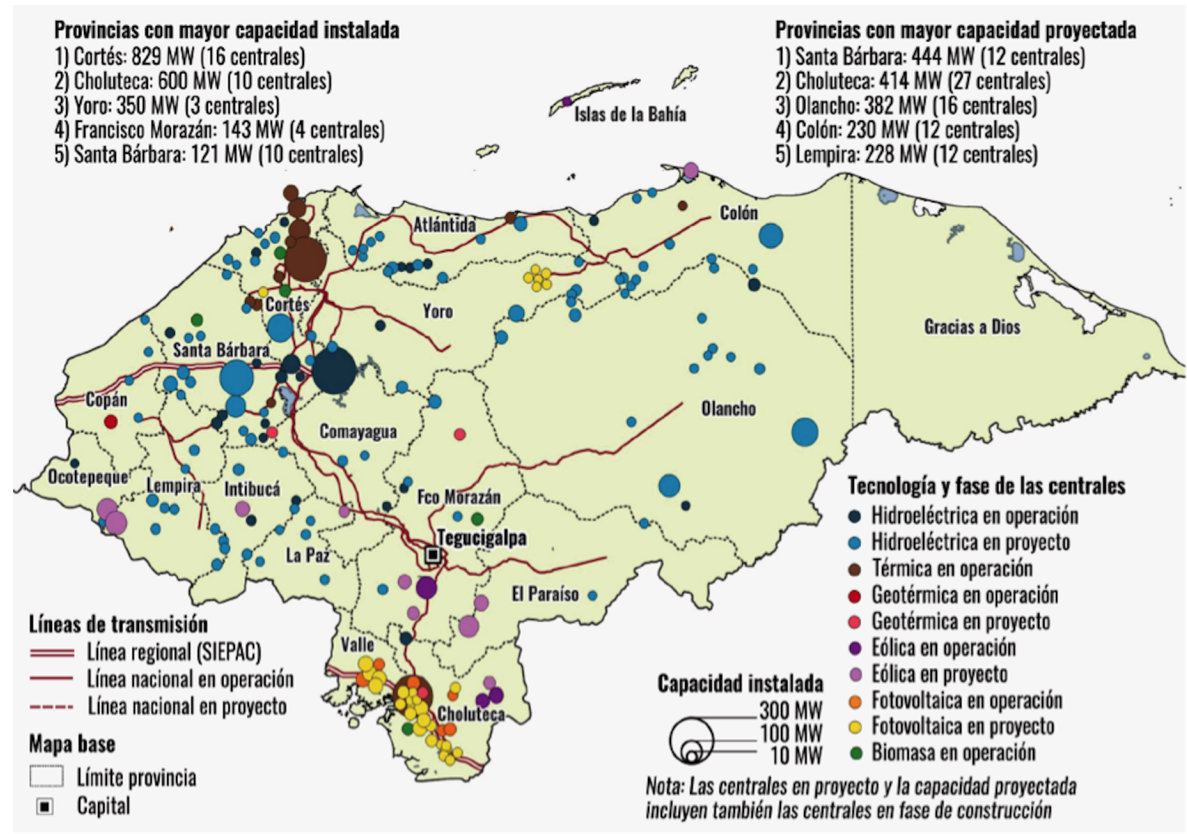

Figura 3. Mapa de proyectos de generación eléctrica por tecnología y capacidad en Honduras. El mapa muestra la localización de las plantas de generación hidroeléctrica, biomasa, geotérmica, eólica, fotovoltáica y térmicas convencionales, de acuerdo a su fase (proyecto, operación o construcción). Fuente: Elaboración propia, descargable en el geovisualizador referido, en Geocomunes (2019c).

Figure 3. Geographic distribution of power plants located at the different departments of Honduras. The map shows hydroelectric, biomass, geothermal, wind, photovoltaic, and fossil-fuel power plants and its status. Source: Prepared by the authors based on the georeferenced databases of this research. Downloadable map from Geocomunes (2019c)

mismo, esta investigación generó y recopiló información espacial sobre proyectos asociados al uso de esta energía (líneas de transmisión nacionales, línea de transmisión del SIEPAC, proyectos mineros, concesiones mineras, zonas francas, etc).

Las particularidades de la información georreferenciada y de los datos que aportan pueden ser consultados en la tabla $1 \mathrm{y}$ en la tabla 4.

\section{Diseño del mapa}

Mientras que la investigación tiene como uno de sus fines la difusión de la información, tanto en sectores académicos especializados, como en sectores no especializados; el diseño del mapa priorizó la simplicidad así como la visualización de cualidades consideradas estratégicas de los proyectos, como fase, tamaño (capacidad de la central), y si está o no conectada al SIEPAC. La interfaz del visualizador permite activar y desactivar la información del mapa mediante un menú de capas agrupadas por categorías (generación eléctrica; transmisión de energía, insumos y mercancías; megaproyectos extractivos, agroindustriales e industria de la transformación; y capas base), en el que se muestra la leyenda o simbología de cada cobertura. Para lograr una comunicación efectiva de la información desarrollada en esta investigación se diseñaron dos mapas por país, uno de ellos mostrando la capacidad de las centrales y su tecnología, y el segundo integrando al análisis más complejidad al mos-

Tabla 3: Financiamiento de algunos bancos multilaterales de desarrollo para proyectos de generación eléctrica en Centroamérica con datos en millones de dólares. Fuente: Elaboración propia con base en la base de datos georreferenciada de esta investigación. Para consultar todos los financamientos de bancos que se lograron ubicar con posibilidad de filtro por banco o proyecto, ver el visualizador referido (Geocomunes, 2019b).

Table 3. Funding in millions of dollars from multilateral development banks for power plant generation projects in Central America. Prepared by the authors based on the georeferenced databases of this research. All information is available in Geocomunes (2019b), and can be refined by country, bank or project.

\begin{tabular}{|c|c|c|c|c|c|c|}
\hline \multirow[t]{2}{*}{ País } & \multicolumn{2}{|c|}{ BCIE } & \multicolumn{2}{|c|}{ BID } & \multicolumn{2}{|c|}{ BM } \\
\hline & Núm. & Monto & Núm. & Monto & Núm. & Monto \\
\hline Guatemala & 4 & 497 & 6 & 260 & 10 & 319 \\
\hline Nicaragua & 7 & 536 & 6 & 157 & 4 & 186 \\
\hline El Salvador & 4 & 420 & 3 & 284 & 4 & 97 \\
\hline Total & 31 & 2,489 & 34 & 2,450 & 41 & 1,722 \\
\hline
\end{tabular}


trar otros proyectos asociados con la transmisión, uso y posible aprovechamiento de esta energía en la región.

\section{Conclusiones}

Este trabajo muestra la construcción de las bases de datos georreferenciadas y el geovisualizador resultado de una primera investigación sobre los proyectos de generación eléctrica en los países que forman parte del SIEPAC. Los ejes de esta investigación se definieron en conjunto con organizaciones sociales, y principalmente con el Movimiento de Afectados por Represas (MER), quién tenía como principales necesidades el poder contar con un mapeo completo de las centrales existentes y proyectadas, con los actores relacionados (empresas y bancos), así como de otros megaproyectos relacionados que también son considerados como amenazas a los territorios y comunidades de la región (minería, agroindustria, corredores logísticos, etcétera). Así, esta investigación es el resultado de un proyecto de colaboración más amplio encaminado hacia la construcción de capacidades de investigación, de colaboración, de monitoreo, de análisis y de argumentación que busca sumar fuerzas propositivas y científicas al escenario social actual. Los resultados del trabajo de búsqueda y rastreo de la presente información, alimentan una base de datos georeferenciada accesible en internet para su uso, para la visualización de las centrales, los textos de análisis y las tablas completas. Las características de los visualizadores en línea de esta información, abren la posibilidad de compartir libremente las bases, así como generar procesos colectivos de participación en la actualización, revisión y corrección de la información, algo especialmente necesario para analizar procesos tan dinámicos como el abordado en esta investigación. La construcción colectiva de este tipo de bases por medio de tecnologías de compartición de datos vectoriales, potencian la escala de participación en procesos de construcción de conocimiento para el análisis y la participación del conjunto de la sociedad organizada. El hecho de que la parte metodológica de construcción de la información esté documentada, las capas y el mapa interactivo realizado se hayan hecho usando software libre y códigos abiertos, y toda la información sea visible y descargable sin restricción alguna, permite que esta iniciativa pueda enriquecerse de manera colaborativa, y constituye un ejemplo técnico y metodológico que podría aplicarse en otras situaciones de colaboración entre la academia y la sociedad civil.

En este sentido, reconocemos que la presente investigación contiene límites, ya que a pesar de estar concebido como parte de un proceso de colaboración entre academia y sociedad civil, la información que presenta no incluye de manera explícita en su mapeo a los sujetos que cuestionan estos proyectos (opositores, afectados directos e indirectos de éstos, etcétera). Esta determinación que, como se ha dicho, fue definida inicialmente como un enfoque en donde se mapea la infraestructura del capital y no a los conflictos resultantes de estos proyectos, corresponde a una discusión colectiva de GeoComunes con las redes de organizaciones sociales con las que trabaja. Sin embargo, como colectivo también somos conscientes del límite que esto puede representar para la interpretación que pueda darse del problema a través del uso de esta información. El riesgo de replicar lecturas donde los sujetos no hegemónicos y sus diversas territorialidades sean invisibilizadas, es alto y grave porque en lugar de aportar información y conocimiento para la transformación social, puede reproducir la dinámica que sistemáticamente reduce y niega proyectos sociales y civilizatorios "alternativos".

Por esta razón, la colaboración y enriquecimiento participativo de la presente base georreferenciada y del visualizador es aún más importante. Así mismo, como colectivo, GeoComunes está ahora desarrollando una colaboración con el proyecto EJAtlas mencionado en este artículo. Dicha colaboración tiene por objetivo ir trabajando una propuesta de mapas interactivos que pueden cruzar los datos espaciales que desarrolla GeoComunes sobre proyectos y el capital involucrado, con los datos espaciales que desarrolla el EJAtlas sobre conflictos. Uno de los primeros productos que resultará de esta colaboración será un mapa interactivo de América Latina sobre proyectos hidroeléctricos y conflictos relacionados próximo a presentar.

\section{Agradecimientos}

Al Colectivo GeoComunes y al Movimiento de Afectados por Represas en América Latina (MAR) por su confianza, retroalimentación y el apoyo mostrado en la construcción de este proyecto conjunto. A la fundación Rosa Luxemburg Stiftung por su amplia solidaridad y los recursos otorgados para realizar esta investigación. Nota sobre el crédito de los autores: Susana Isabel Velázquez Quesada, diseñó el proyecto, realizó la investigación, los textos y la cartografía; Yannick Deniau, diseñó el proyecto, realizó la investigación, los textos y la cartografía; Luis Fernando Pérez Macías, diseñó el proyecto, realizó la investigación, los textos y la cartografía; Iván Alejandro Martínez Zazueta, realizó el geovisualizador.

\section{Referencias}

Autoridad Nacional de los Servicios Públicos (ASEP), sin fecha. Resoluciones Electricidad (consulta en línea). Gobierno de la República de Panamá, <https://www.asep.gob.pa/?category_name=resolucioneselectricidad\&post_types $=$ post $>$, consulta: 15 de abril de 2019.

Autoridad Reguladora de los Servicios Públicos (ARESEP), sin fecha. Información regulatoria (información en línea). Costa Rica, https://aresep.go.cr/operadores/tramites-operadores>, consulta: 15 de abril de 2019.

Comisión Regional de Interconexión Eléctrica (CRIE), 2005. Reglamento del Mercado Eléctrico Regional (RMER) (en línea). $<$ http://crie.org.gt/wp/rmer-reglamento-mercado-electrico-regional/>, consulta: 15 de abril de 2019.

Comisión Reguladora de Energía Eléctrica (CREE), sin fecha. Regulación. Instituto de Acceso a la Información Pública, Honduras, $<$ https://portalunico.iaip.gob.hn/portal/index.php?portal=444> , consulta: 15 de abril de 2019.

Consejo Nacional de Energía (CNE), sin fecha. Energías Renovables (información en línea). Gobierno de El Salvador, <https://www.cne.gob.sv/?page_id=459>, consulta: 15 de abril de 2019. 


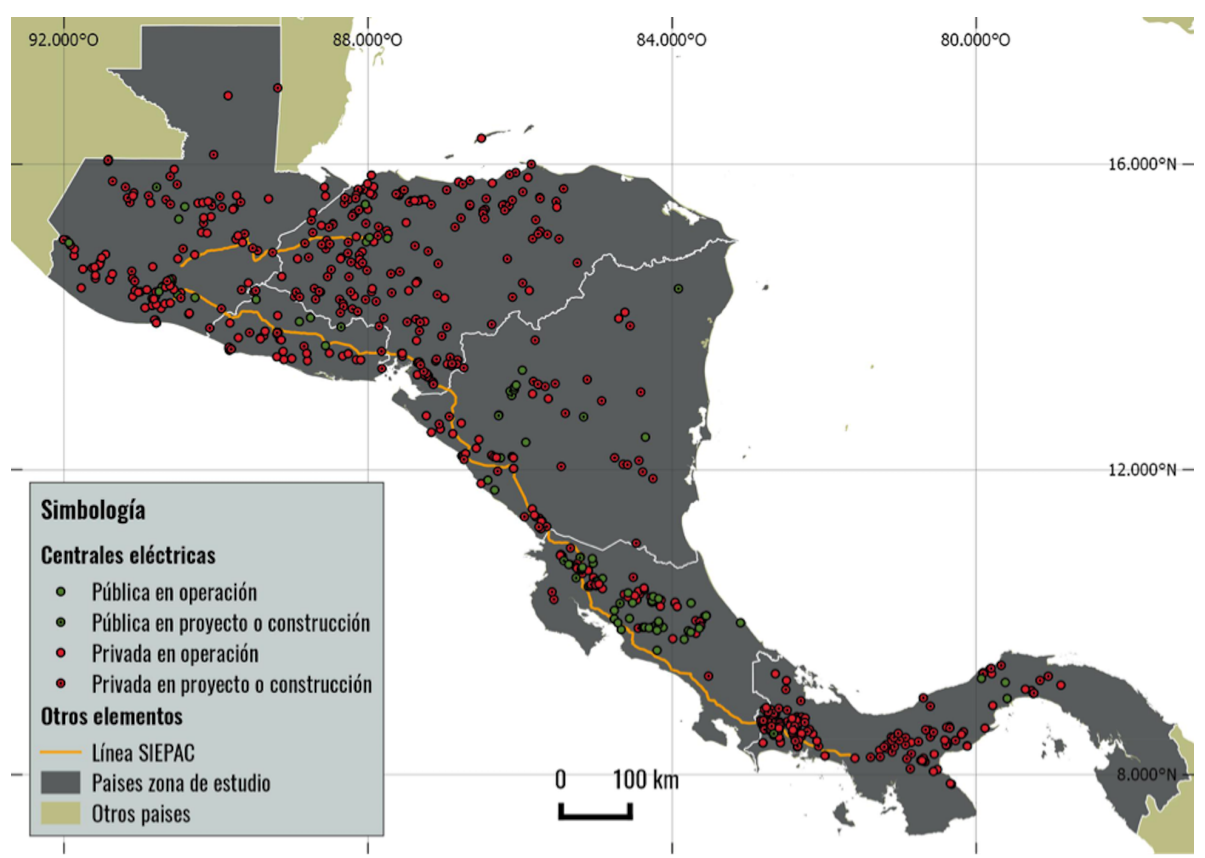

Figura 4. Mapa de proyectos de generación eléctrica por sector público o privado, y de acuerdo con la fase del proyecto (operación, proyecto o contrucción), así como otros elmentos significativos, como la línea de transmisión del Sistema de Interconexión Eléctrica para América Central (SIEPAC), y la delimitación de los países del área de estudio. Fuente: Elaboración propia.

Figure 4. Geographic distribution of power plants (public and private) located in Central America. The map also shows the Central American Electrical Interconnection System (SIEPAC). Source: Prepared by the authors based on the georeferenced databases of this research.

Crampton J. W., Krygier J., 2006. An Introduction to Critical Cartography (en línea). ACME: An International Journal for Critical Geographies 4 (1), 1133. <https://acme-journal.org/index.php/acme/article/view/723>, consulta: 10 de abril 2019.

Echavarría C., Jesurun-Clements N., Mercado-Díaz J. E., Trujillo C., 2017. Integración eléctrica centroamericana: Génesis, beneficios y prospectiva del Proyecto SIEPAC, Sistema de Interconexión Eléctrica de los Países de América Central (en línea). Banco Interamericano de Desarrollo (BID), <https://publications.iadb.org/es/publicacion/13974/integracionelectrica-centroamericana-genesis-beneficios-y-prospectiva-del $>$, consulta: 15 de abril de 2019.

Empresa de Transmisión Eléctrica Panameña (ETESA), sin fecha. Compras de Energía y/o Potencia (consulta en línea). Panamá, < https://www.etesa.com.pa/compras.php>, consulta: 15 de abril de 2019.

Empresa Propietaria de la Red (EPR), 2014. Informe General. Antecedentes, estado actual y perspectivas del Sistema de Interconexión eléctrica para los países de América Central (SIEPAC) (en línea). $<\mathrm{http}: / /$ www.eprsiepac.com/pdf/informe_general_linea_siepac_dic13.pdf $>$, consulta: 10 de febrero 2019.

Environmental Justice Atlas (EjatAtlas), sin fecha. World Map (consulta interactiva). <https://ejatlas.org/>, consulta el 10 de abril de 2019.

Geocomunes, 2019a. Expansión de proyectos eléctricos en Centroamérica. El desarrollo de un sistema eléctrico regional fuera del control de los pueblos (en línea). México, $<$ http://geocomunes.org/Analisis_PDF/CuadernilloCA $>$, consulta: 15 de abril de 2019.

Geocomunes, 2019b. Lista de Financiamientos. En Expansión de proyectos eléctricos en Centroamérica. El desarrollo de un sistema eléctrico regional fuera del control de los pueblos (en línea). México, $<$ http://geocomunes.org/Analisis_PDF/Detalles_financiadores.xls>, consulta: 15 de abril de 2019.

Geocomunes, 2019c. Mapa de proyectos eléctricos en Honduras (en línea). México, <http://geocomunes.org/Analisis_PDF/Honduras Lectura.pdf >, consulta: 15 de abril de 2019 .

Gobierno de Honduras, sin fecha. Diario Oficial de la Republica de
Honduras, La Gaceta. Empresa Nacional de Artes Gráficas (ENAG), $<$ www.lagaceta.hn $>$, consulta: 15 de abril de 2019 .

Instituto Costarricense de Electricidad, sin fecha. Información regulatoria (información en línea). Costa Rica, ASESEP, < https://www.grupoice.com/wps/portal>, consulta: 15 de abril de 2019.

Instituto Nicaragüense de Energía (INE), sin fecha. Electricidad (información en línea). Gobierno de Nicaragua, < https://www.ine.gob.ni/index.php/electricidad/>, consulta: 15 de abril de 2019.

Ministerio de Energía y Minas (MEM), sin fechaa. Electricidad (información en línea). Guatemala, < http://www.mem.gob.gt/energia/electricidad/>, consulta 15 de abril de 2019.

Ministerio de Energía y Minas (MEM), sin fechab. Electricidad y Recursos Renovables (información en línea). Gobierno de Nicaragua, < http://www.mem.gob.ni/?page_id=3274>, consulta: 15 de abril de 2019.

Osorno-Covarrubias J., Couturier S., López-Quiroz P., Ferrari L., MartinezZazueta I., 2018. TERRA DIGITALIS: Diseño e implementación de una revista internacional interactiva de mapas arbitrados. Geofocus-Revista Internacional de Ciencia y Tecnologia de la Informacion Geografica, (22), 115-139. http://dx.doi.org/10.21138/GF.555.

Osorno-Covarrubias J., Couturier S., Ricardez M., 2015. El rol de la Geografía y sus hibridaciones recientes frente a la crisis de sustentabilidad global. Boletin de la asociacion de geografos españoles, (69), 93-112. http://dx.doi.org/10.21138/bage.1891.

Rojas-Navarrete M., 2018. Estadísticas de producción de electricidad de los países del Sistema de la Integración Centroamericana (SICA): Datos preliminares a 2017 (en línea). Comisión Económica por América Latina y el Caribe (CEPAL), $<$ http://repositorio.cepal.org/bitstream/handle/11362/44358/1/S1801216_es. pdf $>$, consulta: 15 de abril de 2019.

Sandá-Mera A., 2014. El negocio de la energía eléctrica en Centroamérica y El Salvador: alianzas de integración regional y proyectos que profundizan la dominación de las corporaciones transnacionales. El caso de la central hidroeléctrica El Chaparral (en línea). Observatorio de Multinacionales de América Latina (OMAL), Centro de Investigación sobre Inversión y Comer- 
Tabla 4: Información de otros elementos vectoriales georreferenciados en esta investigación y que pueden ser visualizados y descargados en el geovisualizador mencionado. Fuente: Elaboración propia.

Table 4. Detailed information about georeferenced layers included in the geo-visualizer. Source: Prepared by the authors based on the georeferenced databases of this research.

\begin{tabular}{|c|c|c|c|}
\hline Capa de información & $\begin{array}{l}\text { Escala de la } \\
\text { información }\end{array}$ & $\begin{array}{c}\text { Tipo de información } \\
\text { vectorial }\end{array}$ & Cualidades de los elementos \\
\hline \multicolumn{4}{|c|}{ Proyectos de transmisión de energía } \\
\hline $\begin{array}{c}\text { Líneas de SIEPAC } \\
\text { Líneas de transmisión nacionales }\end{array}$ & $\begin{array}{l}\text { Regional } \\
\text { Regional }\end{array}$ & $\begin{array}{l}\text { Líneas } \\
\text { Líneas }\end{array}$ & $\begin{array}{l}\text { Sin caracterización de la información } \\
\text { Se desglosa si está en construcción, proyecto u opera- } \\
\text { ción. }\end{array}$ \\
\hline \multicolumn{4}{|c|}{ Proyectos de transmisión de mercancías e insumos } \\
\hline $\begin{array}{c}\text { Puertos } \\
\text { Red Integral de Carreteras de } \\
\text { América Central } \\
\text { Corredores multimodales } \\
\text { interoceánicos } \\
\end{array}$ & $\begin{array}{l}\text { Regional } \\
\text { Regional } \\
\text { Regional }\end{array}$ & $\begin{array}{l}\text { Puntos } \\
\text { Líneas } \\
\text { Líneas }\end{array}$ & $\begin{array}{l}\text { Se desglosa si están en operación o en proyecto. } \\
\text { Sin caracterización de la información } \\
\text { Se desglosa en proyecto de canal, corredor seco existen- } \\
\text { te o canal. }\end{array}$ \\
\hline \multicolumn{4}{|c|}{ Proyectos Extractivos } \\
\hline $\begin{array}{l}\text { Zona de cultivo de palma africana en } \\
\text { Centroamérica }\end{array}$ & Regional & Polígonos & Sin caracterización de la información \\
\hline $\begin{array}{l}\text { Zonas de monocultivo de piña en } \\
\text { Costa Rica }\end{array}$ & Nacional (Costa Rica) & Polígonos & Sin caracterización de la información \\
\hline $\begin{array}{c}\text { Zonas de cultivo de caña de azúcar } \\
\text { en Guatemala }\end{array}$ & Nacional (Guatemala) & Polígonos & Sin caracterización de la información \\
\hline
\end{tabular}

Industria y proyectos asociados a la transformación de materias primas

\begin{tabular}{|c|c|c|c|}
\hline Zonas Francas & Regional & Puntos & $\begin{array}{l}\text { Se desglosa en función de su estado: en operación o en } \\
\text { proyecto. }\end{array}$ \\
\hline $\begin{array}{l}\text { Proyecto de Zonas Económicas } \\
\text { Especiales en El Salvador }\end{array}$ & Nacional (El Salvador) & Polígonos & Sin caracterización de la información \\
\hline \multicolumn{4}{|c|}{ Capas Base } \\
\hline Ríos & Regional & Líneas & Sin caracterización de la información \\
\hline
\end{tabular}

cio $(\mathrm{CEICOM}),<$ omal.info/IMG/pdf/negocio_electricidad.pdf $>$, consulta: 15 de abril de 2019 .

Superintendencia General de Electricidad y Telecomunicaciones (SIGET), sin fecha. Electricidad: Documentos (información en línea). El Salvador, <https://www.siget.gob.sv/temas/electricidad/documentos/>, consulta: 15 de abril de 2019.
This article accompanies the following material:

HTML:

DOI: $10.22201 /$ igg.25940694.2019.1.57.119

External interactive map: DOI: 10.22201/igg.25940694.2019.1.57.120 